$\mathrm{UCTP}-3 / 97$

VPI-IPPAP-97-3

hep-ph/9702440

\title{
ACD Estimation of the $S$-parameter Revisited
}

\author{
S. R. Ignjatović ${ }^{1)}$, T. Takeuchi ${ }^{2}$, and L. C. R. Wijewardhana ${ }^{1)}$ \\ 1) Department of Physics, University of Cincinnati, Cincinnati, OH 45221 \\ ${ }^{2)}$ Institute for Particle Physics and Astrophysics, \\ Physics Department, Virginia Tech, Blacksburg, VA 24061-0435
}

\begin{abstract}
The analytic continuation by duality (ACD) technique has been used to estimate the electroweak $S$ parameter in technicolor models. In this letter, we investigate the reliability of this method by applying it to some toy models with known spectra. We find that in most instances the technique cannot be trusted to give a reliable result.
\end{abstract}

$\mathrm{UCTP}-3 / 97$

VPI-IPPAP-97-3

hep-ph/9702440

February 1997 


\section{Introduction}

There is no need to emphasize the importance of finding reliable non-perturbative calculational techniques that can be applied to strongly interacting theories such as technicolor. Without such methods, strongly interacting theories can never be tested since no quantitative predictions that can be compared directly with experiment can be made.

The analytic continuation by duality (ACD) technique, which was first developed in Ref. [1], has been proposed in Ref. [2] as a potentially reliable way to compute the oblique correction parameter $S$ for technicolor theories. The advantage of the ACD technique was that it could be applied to both QCD-like and walking technicolor [3] theories whereas the dispersion relation technique used by Peskin and one of us in Ref. [4] could only be applied to the former. Furthermore, the ACD estimate of $S$ for walking technicolor implied that walking dynamics could render $S$ negative, making it compatible with the current experimental limit. This was in contrast to the result of Harada and Yoshida [5] who used the Bethe-Salpeter equation approach to conclude that $S$ was positive even for walking theories.

However, the robustness of the ACD technique has been questioned in the literature [6]. In this letter, we further investigate the reliability of the ACD technique. In section 2, we review the definition of the $S$ parameter and explain the ACD technique. In section 3, we apply the ACD technique to the perturbative spectral function that represents the contribution of a degenerate doublet of heavy fermions to see if the well known result $1 / 6 \pi$ could be reproduced. In section 4 , we apply the ACD technique to a model function which has an imaginary part more representative of the actual QCD spectrum. Section 5 concludes.

\section{The ACD technique}

The ACD technique consists of using the analyticity of the vacuum polarization function to convert the dispersion integral for $\mathrm{S}$, which is an integral along the real $s$ axis, into an integral around a large circle in the complex $s$ plane. The value of the integral on the circle is then estimated by the Operator Product Expansion (OPE) [7].

We denote by $\Pi_{\mathrm{XY}}$ the $g^{\mu \nu}$ part of the vacuum polarization tensor between 
two currents $J_{\mathrm{X}}^{\mu}$ and $J_{\mathrm{Y}}^{\nu}$ :

$$
i g^{\mu \nu} \Pi_{\mathrm{XY}}(s)+\left(q^{\mu} q^{\nu} \text { term }\right)=\int d^{4} x e^{i q \cdot x}\left\langle J_{\mathrm{X}}^{\mu}(x) J_{\mathrm{Y}}^{\nu}(0)\right\rangle, \quad s=q^{2} .
$$

The $S$ parameter is defined in Ref. [四] as

$$
S=-4 \pi\left[\Pi_{\mathrm{VV}}^{\prime}(0)-\Pi_{\mathrm{AA}}^{\prime}(0)\right]
$$

where the subscripts $V$ and $A$ denote the neutral isospin-1 vector and axialvector currents, respectively, and the prime denotes a derivative with respect to $s$.

For latter convenience, we define the functions $\mathcal{F}_{\mathrm{V}}(s)$ and $\mathcal{F}_{\mathrm{A}}(s)$ as

$$
\begin{aligned}
& \Pi_{\mathrm{VV}}(s) \equiv s \mathcal{F}_{\mathrm{V}}(s) \\
& \Pi_{\mathrm{AA}}(s) \equiv \Pi_{\mathrm{AA}}(0)+s \mathcal{F}_{\mathrm{A}}(s)
\end{aligned}
$$

and the following shorthand notation for the difference between $\mathcal{F}_{\mathrm{V}}(s)$ and $\mathcal{F}_{\mathrm{A}}(s)$ :

$$
\mathcal{F}(s) \equiv \mathcal{F}_{\mathrm{V}}(s)-\mathcal{F}_{\mathrm{A}}(s)
$$

Then,

$$
S=-4 \pi\left[\mathcal{F}_{\mathrm{V}}(0)-\mathcal{F}_{\mathrm{A}}(0)\right]=-4 \pi \mathcal{F}(0) .
$$

Note that our notation is slightly different from either Ref. [2] or Ref. [4] so care is necessary when comparing formulae.

The function $\mathcal{F}(s)=\mathcal{F}_{\mathrm{V}}(s)-\mathcal{F}_{\mathrm{A}}(s)$ is analytic in the entire complex $s$ plane except for a branch cut along the positive real $s$ axis starting from the lowest particle threshold contributing to $\mathcal{F}(s)$. Therefore, applying Cauchy's theorem to the contour $C$ shown in Fig. 1, we find

$$
\begin{aligned}
S & =-4 \pi \mathcal{F}(0) \\
& =-4 \pi\left[\frac{1}{\pi} \int_{s_{0}}^{R} d s \frac{\operatorname{Im} \mathcal{F}(s)}{s}+\frac{1}{2 \pi i} \oint_{|s|=R} d s \frac{\mathcal{F}(s)}{s}\right] .
\end{aligned}
$$

If the radius of the contour $R$ is taken to infinity, the integral around the circle at $|s|=R$ vanishes and we obtain the dispersion relation

$$
S=-4 \int_{s_{0}}^{R} d s \frac{\operatorname{Im} \mathcal{F}(s)}{s},
$$




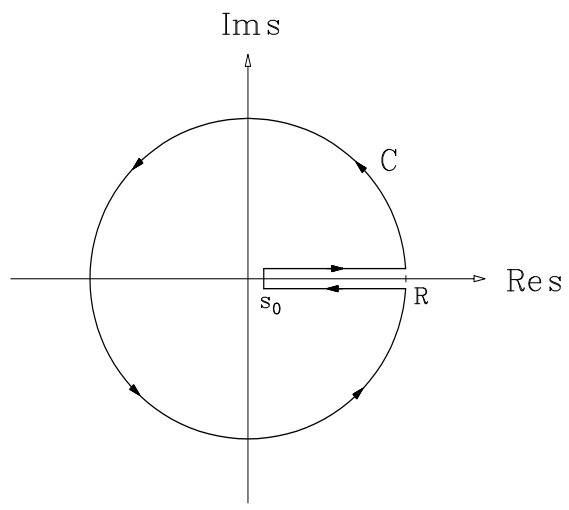

Figure 1: The contour $C$ which avoids the branch cut along the real $s$-axis.

which was used in Ref. [4 to calculate $S$. However, the dispersion relation approach requires the knowledge of $\operatorname{Im} \mathcal{F}(s)$ along the real $s$ axis which is only available for QCD-like theories.

The basic idea of the ACD technique, on the other hand, is to approximate the kernel $1 / s$ by a polynomial

$$
\frac{1}{s} \approx p_{N}(s)=\sum_{n=0}^{N} a_{n}(N) s^{n}, \quad s \in\left[s_{0}, R\right]
$$

and use it to make the integral along the real $s$ axis vanish instead. Applying Cauchy's theorem to the product $p_{N}(s) \mathcal{F}(s)$ over the same contour $C$ yields

$$
0=-4 \pi\left[\frac{1}{\pi} \int_{s_{0}}^{R} d s p_{N}(s) \operatorname{Im} \mathcal{F}(s)+\frac{1}{2 \pi i} \oint_{|s|=R} d s p_{N}(s) \mathcal{F}(s)\right] .
$$

Subtracting Eq. (2.2) from Eq. (2.1), we obtain

$$
S=S_{N}+\Delta_{\text {fit }},
$$

where

$$
S_{N} \equiv-\frac{2}{i} \oint_{|s|=R} d s\left[\frac{1}{s}-p_{N}(s)\right] \mathcal{F}(s),
$$




$$
\Delta_{\mathrm{fit}} \equiv-4 \int_{s_{0}}^{R} d s\left[\frac{1}{s}-p_{N}(s)\right] \operatorname{Im} \mathcal{F}(s) .
$$

For sufficiently large $N$, we can expect $\Delta_{\text {fit }}$ to be negligibly small. In fact, it converges to zero in the limit $N \rightarrow \infty$ (though how quickly the convergence occurs depends on the interval $\left.\left[s_{0}, R\right]\right)$. We can therefore neglect it and approximate $S$ with $S_{N}$ which is an integral around the circle $|s|=R$ only. We call $\Delta_{\text {fit }}$ the fit error.

In fitting $p_{N}(s)$ to $1 / s$, the $s$-coefficients $a_{n}(N)$ 's are determined so that some norm of the difference function

$$
d(s)=\frac{1}{s}-p_{N}(s)
$$

on $\left[s_{0}, R\right]$ is minimized. It is usually an $L_{p}$ norm:

$$
\|d\| \equiv\left[\int_{s_{0}}^{R}|d(s)|^{p} w(s) d s\right]^{1 / p},
$$

where $w(s)$ is some weight function. Obviously, different choices of $p$ and $w(s)$ will lead to different fitting coefficients $a_{n}(N)$, thus different values for $S_{N}$ and $\Delta_{\text {fit }}$. Since this ambiguity has not been explored in the literature on ACD, we have done a detailed analysis that will be presented elsewhere [B] The fitting routine dependence proved to be mild.

If the radius of the contour $R$ is taken to be sufficiently large, the function $\mathcal{F}(s)$ can be approximated on $|s|=R$ by a large momentum expansion:

$$
\mathcal{F}(s) \approx \sum_{m=1}^{M} \frac{h_{m}(s)}{s^{m}} .
$$

This expression is obtained by analytically continuing the operator product expansion $(\mathrm{OPE})$ of $\mathcal{F}(s)$ [7], which is available for both QCD-like and walking technicolor theories, from the deep Euclidean region $s \ll 0$. Therefore, we can write

$$
S_{N}=S_{N, M}+\Delta_{\text {tr }},
$$

where

$$
S_{N, M} \equiv-\frac{2}{i} \oint_{|s|=R} d s\left[\frac{1}{s}-p_{N}(s)\right] \sum_{m=1}^{M} \frac{h_{m}(s)}{s^{m}},
$$




$$
\Delta_{\mathrm{tr}} \equiv-\frac{2}{i} \oint_{|s|=R} d s\left[\frac{1}{s}-p_{N}(s)\right]\left[\mathcal{F}(s)-\sum_{m=1}^{M} \frac{h_{m}(s)}{s^{m}}\right],
$$

and approximate $S_{N}$ with $S_{N, M}$. The neglected term $\Delta_{\text {tr }}$ is called the truncation error. It is actually not clear whether the truncation error will go to zero in the limit $M \rightarrow \infty$ since Eq. (2.3) may be an asymptotic series and not a convergent one. If it is asymptotic, taking $M$ to be larger than an optimum value may worsen the approximation. However, $M$ must be taken to be larger than $N$ to suppress $\Delta_{\text {tr }}$ with inverse powers of $R$. If the series is convergent, the convergence may not be particularly fast requiring a very large $M$ and/or $R$ to suppress $\Delta_{\text {tr }}$. Some of these points will be discussed in the following, but a more complete treatment will be given in Ref. [8].

If the $s$-dependence of the expansion coefficients in Eq. (2.3) is "negligibly weak" then the approximation can be taken one step further and the $s^{-}$ dependence of the $h_{m}(s)$ 's dropped, i.e.

$$
h_{m}(s) \approx h_{m}(-R) \equiv \hat{h}_{m}
$$

(One must keep in mind that this is a very dangerous approximation to make since the analytic structure of the integrand will be completely altered no matter how "weak" the $s$-dependence may be: the imaginary part of $\mathcal{F}(s)$ will be reduced to derivatives of $\delta$-functions at the origin.) Therefore,

$$
S_{N, M}=S_{\mathrm{ACD}}+\Delta_{\mathrm{AC}}
$$

where

$$
\begin{aligned}
S_{\mathrm{ACD}} & \equiv-\frac{2}{i} \oint_{|s|=R} d s\left[\frac{1}{s}-p_{N}(s)\right] \sum_{m=1}^{M} \frac{\hat{h}_{m}}{s^{m}} \\
\Delta_{\mathrm{AC}} & \equiv-\frac{2}{i} \oint_{|s|=R} d s\left[\frac{1}{s}-p_{N}(s)\right] \sum_{m=1}^{M} \frac{h_{m}(s)-\hat{h}_{m}}{s^{M}}
\end{aligned}
$$

and we can approximate $S_{N, M}$ with $S_{A C D}$. Unfortunately, this is a tautological statement: The only way one can conclude that the $s$-dependence was "negligibly weak" is to calculate $\Delta_{\mathrm{AC}}$ and find that it is indeed suppressed. However, it is possible to give the following handwaving argument that $\Delta_{\mathrm{AC}}$ would be generically small: The difference $h_{m}(s)-\hat{h}_{m}$ in the integrand of $\Delta_{\mathrm{AC}}$ can be expected to be smallest in the deep Euclidean region and most 
pronounced in the vicinity of the positive real $s$ axis. But since that is where the difference $1 / s-p_{N}(s)$ is approximately zero, the contribution of this large difference to $\Delta_{\mathrm{AC}}$ with be highly suppressed. Thus:

$$
S \approx S_{\mathrm{ACD}}
$$

In this approximation, the integral for $S_{\mathrm{ACD}}$ will only pick up the residues of the single poles inside the integration contour and we find,

$$
S_{\mathrm{ACD}}=4 \pi \sum_{n=0}^{\min \{N, M-1\}} a_{n}(N) \hat{h}_{n+1} .
$$

We will call $\Delta_{\mathrm{AC}}$ the analytical continuation error.

To summarize, the ACD technique uses the relation

$$
S=S_{\mathrm{ACD}}+\Delta_{\mathrm{AC}}+\Delta_{\mathrm{tr}}+\Delta_{\mathrm{fit}},
$$

and assumes that all three types of error can be neglected and approximates $S$ with $S_{\mathrm{ACD}}$. Therefore, the question of whether the ACD technique is reliable or not can be translated to the question of whether all these errors are under control or not.

In actual applications of the ACD technique, there is an additional source of error due to the fact that the expansion coefficients $\hat{h}_{m}$ are only known approximately. This is because the $\hat{h}_{m}$ 's are products of Wilson coefficients and condensates of operators. The Wilson coefficients can only be calculated perturbatively, and only a very limited number of them are known. The condensates are even less known and only a handful of them have been determined phenomenologically. As a result, the numerical uncertainty on $\hat{h}_{m}$ increases with $m$ so increasing $N$ and $M$ does not necessarily improve the final estimate of $S_{\mathrm{ACD}}$ since it may be swamped by the numerical uncertainty of the higher order terms one must include. A common strategy in the literature is to try to choose optimum values of $N$ and $M$ such that the fit and truncation errors are suppressed, but the numerical uncertainty is not yet too large. The procedure essentially consists of the minimization of a 'total' error. However, it is very difficult to define the total error since it involves a mathematically well defined error and, on the other hand, an error which reflects our ignorance. The problem itself is ill-posed and difficult to treat with any level of rigor. 
Table 1: $S_{\mathrm{ACD}}$ and the fit, truncation, and analytical continuation errors for the perturbative model function. The cutoffs are $\left[s_{0}, R\right]=\left[4 \mathrm{~m}^{2}, 25 \mathrm{~m}^{2}\right]$, and the fit routine was the least square fit. The exact value of $S$ is $1 / 6 \pi=0.0531$.

\begin{tabular}{cccccr}
\hline \hline$N$ & $M$ & $S_{\mathrm{ACD}}$ & $S_{N, M}=S_{\mathrm{ACD}}+\Delta_{\mathrm{AC}}$ & $\Delta_{\mathrm{fit}}$ & \multicolumn{1}{c}{$\Delta_{\mathrm{tr}}$} \\
\hline \hline 3 & 2 & 0.2930 & 0.0580 & -0.0002 & -0.0048 \\
& 3 & 0.2883 & 0.0530 & & 0.0002 \\
& 4 & 0.2884 & 0.0532 & & -0.0000 \\
\hline 4 & 2 & 0.4330 & 0.0632 & -0.0001 & -0.0101 \\
& 3 & 0.4203 & 0.0521 & & -0.0010 \\
& 4 & 0.4211 & 0.0532 & & -0.0000 \\
& 5 & 0.4211 & 0.0531 & & 0.0000 \\
\hline 5 & 3 & 0.5731 & 0.0506 & -0.0000 & 0.0025 \\
& 4 & 0.5759 & 0.0533 & & -0.0002 \\
& 5 & 0.5757 & 0.0531 & & 0.0000 \\
& 6 & 0.5757 & 0.0531 & & -0.0000 \\
\hline \hline
\end{tabular}

We will therefore ignore this last type of error in this letter and confine ourselves to the question: if the expansion coefficients of Eq. (2.3) were known exactly, would the ACD technique give the correct result?

\section{The Perturbative Spectral Function}

Now we investigate the reliability of the ACD technique by evaluating $S_{\mathrm{ACD}}$ and the three errors $\Delta_{\text {fit }}, \Delta_{\text {tr }}$, and $\Delta_{\mathrm{AC}}$ in some toy models. First we take the one-loop perturbative contribution of a doublet of heavy fermions to $S$. The contribution to the vacuum polarization coming from this fermion doublet can readily be computed [ [4], and therefore the $S$ parameter can easily be evaluated; its value is $1 / 6 \pi$.

Next, we use the ACD technique to do the same computation. The perturbative spectral function is

$$
\mathcal{F}_{\text {pert }}(s)=\frac{1}{4 \pi^{2}} \frac{m^{2}}{s} \int_{0}^{1} d x \log \left[1-x(1-x) \frac{s}{m^{2}}\right] .
$$


It is analytic in the entire complex $s$ plane except for a branch cut along the positive real $s$ axis which starts from $s=4 m^{2}$. The imaginary part of this function along the cut is given by

$$
\operatorname{Im} \mathcal{F}_{\text {pert }}(s)=-\frac{1}{4 \pi} \frac{m^{2}}{s} \beta \theta\left(s-4 m^{2}\right), \quad \beta=\sqrt{1-\frac{4 m^{2}}{s}} .
$$

The first few terms of the large $s$-expansion of $\mathcal{F}_{\text {pert }}(s)$ are given by

$$
\begin{aligned}
& -4 \pi^{2} \mathcal{F}_{\text {pert }}(s) \\
& =x\left\{-\ln \left(-\frac{1}{x}\right)+2\right\}+x^{2}\left\{2 \ln \left(-\frac{1}{x}\right)+2\right\}+x^{3}\left\{2 \ln \left(-\frac{1}{x}\right)-1\right\}+\ldots,
\end{aligned}
$$

where $x \equiv 4 m^{2} / s$. Using these expressions, we calculated $S_{\mathrm{ACD}}, \Delta_{\mathrm{AC}}, \Delta_{\mathrm{tr}}$, and $\Delta_{\text {fit }}$. The result of our calculations for several values of $N$ and $M$ are shown in Table 1. The fit interval was $\left[s_{0}, R\right]=\left[4 \mathrm{~m}^{2}, 25 \mathrm{~m}^{2}\right]$, and the fit routine was the least square fit.

As is evident from Table 1, the fit and truncation errors are under excellent control and $S_{N, M}$ reproduces the exact value of $S$ accurately already at $N=M=3$. However, the analytic continuation error is not. For the $N=5$ case, for instance, $S_{\mathrm{ACD}}$ is larger than the exact value by more than an order of magnitude. In fact, we find that $S_{\mathrm{ACD}}$ and $\Delta_{\mathrm{AC}}$ diverge as $N \rightarrow \infty$.

We conclude that neglecting the $s$-dependence of the $h_{m}(s)$ 's fails miserably as an approximation. The reason for this can be traced to the fact that even though the difference $1 / s-p_{N}(s)$ converges to zero within its radius of convergence, outside it diverges. Therefore, the handwaving argument we gave in the previous section was wrong: the error induced by the neglect of the $s$-dependence of the $h_{m}(s)$ 's may be suppressed near the real $s$ axis, but it is actually enhanced away from it.

\section{The Breit-Wigner Model}

In light of our result for the perturbative spectral function, the natural question to ask next is whether including the $s$-dependence of the expansion coefficients, i.e. not neglecting $\Delta_{\mathrm{AC}}$, would give an accurate estimate of $S$ for all cases. 


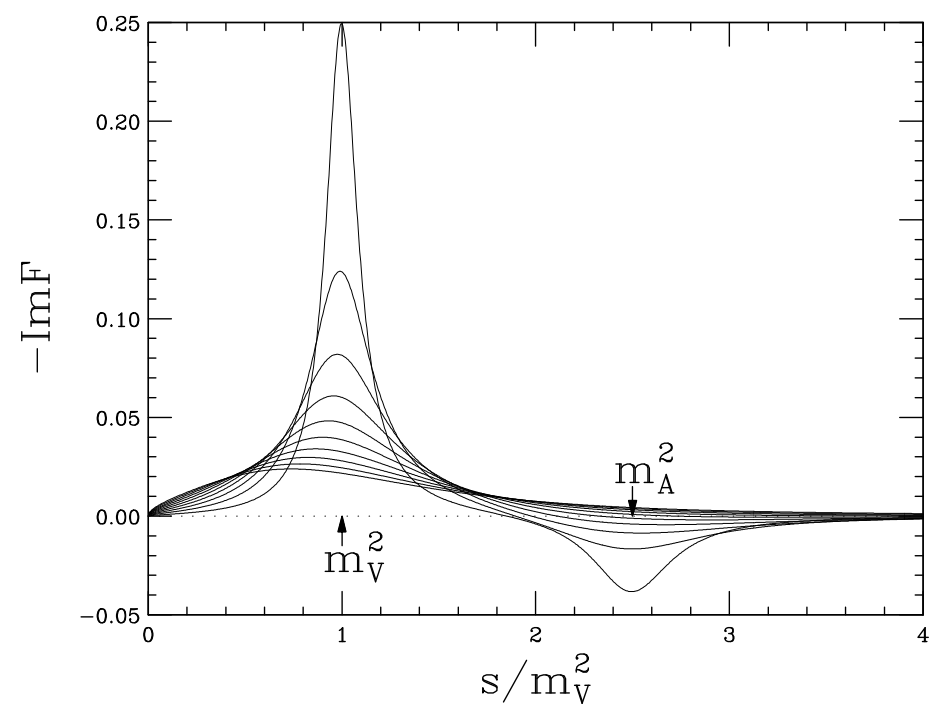

Figure 2: The Spectral Functions for the Breit-Wigner Model. The ratio $\xi \equiv \Gamma_{\mathrm{V}} / m_{\mathrm{V}}=\Gamma_{\mathrm{A}} / m_{\mathrm{A}}$ is varied from $\xi=1.0$ to $\xi=0.1$ at 0.1 intervals with smaller values of $\xi$ corresponding to more prominent peaks. The arrows indicate the positions of the techni $-\rho$ and techni $-a_{1}$ peaks.

To answer this question, we consider the following model:

$$
\begin{aligned}
& \mathcal{F}_{\mathrm{V}}(s)=f_{\mathrm{V}}^{2} \frac{1}{s-m_{\mathrm{V}}^{2}+i \sqrt{s} \Gamma_{\mathrm{V}}}, \\
& \mathcal{F}_{\mathrm{A}}(s)=f_{\mathrm{A}}^{2} \frac{1}{s-m_{\mathrm{A}}^{2}+i \sqrt{s} \Gamma_{\mathrm{A}}} .
\end{aligned}
$$

These functions are analytic in the complex $s$ plane except for a branch cut along the positive real $s$ axis starting from $s=0$. The imaginary part along the cut is of the Breit-Wigner type:

$$
\begin{aligned}
\operatorname{Im} \mathcal{F}_{\mathrm{V}}(s) & =-f_{\mathrm{V}}^{2} \frac{\sqrt{s} \Gamma_{\mathrm{V}}}{\left(s-m_{\mathrm{V}}^{2}\right)^{2}+s \Gamma_{\mathrm{V}}^{2}} \theta(s), \\
\operatorname{Im} \mathcal{F}_{\mathrm{A}}(s) & =-f_{\mathrm{A}}^{2} \frac{\sqrt{s} \Gamma_{\mathrm{A}}}{\left(s-m_{\mathrm{A}}^{2}\right)^{2}+s \Gamma_{\mathrm{A}}^{2}} \theta(s) .
\end{aligned}
$$

We impose the Weinberg sum rules [9] on these functions which leads to 
the constraints'

$$
\begin{aligned}
f_{\mathrm{V}}^{2}-f_{\mathrm{A}}^{2} & =f_{\mathrm{TC}}^{2}, \\
f_{\mathrm{V}}^{2} m_{\mathrm{V}}^{2}-f_{\mathrm{A}}^{2} m_{\mathrm{A}}^{2} & =0 \\
f_{\mathrm{V}}^{2} \Gamma_{\mathrm{V}}^{2}-f_{\mathrm{A}}^{2} \Gamma_{\mathrm{A}}^{2} & =0
\end{aligned}
$$

where $f_{\mathrm{TC}}$ is the technipion decay constant.

The value of $S$ for this model is given by

$$
S=-4 \pi\left[\mathcal{F}_{\mathrm{V}}(0)-\mathcal{F}_{\mathrm{A}}(0)\right]=4 \pi\left[\frac{f_{\mathrm{V}}^{2}}{m_{\mathrm{V}}^{2}}-\frac{f_{\mathrm{A}}^{2}}{m_{\mathrm{A}}^{2}}\right]=4 \pi(1+r) \frac{f_{\mathrm{TC}}^{2}}{m_{\mathrm{V}}^{2}}
$$

where $r \equiv m_{\mathrm{V}}^{2} / m_{\mathrm{A}}^{2}$. We fix $r$ and the ratio $f_{\mathrm{TC}}^{2} / m_{\mathrm{V}}^{2}$ using large $-N$ rescaling from QCD:

$$
\begin{aligned}
\frac{f_{\mathrm{TC}}^{2}}{m_{\mathrm{V}}^{2}} & \approx \frac{f_{\pi}^{2}}{m_{\rho}^{2}} \frac{N_{\mathrm{D}} N_{\mathrm{TC}}}{3} \approx 0.005 N_{\mathrm{D}} N_{\mathrm{TC}} \\
r=\frac{m_{\mathrm{V}}^{2}}{m_{\mathrm{A}}^{2}} & \approx \frac{m_{\rho}^{2}}{m_{a_{1}}^{2}} \approx 0.4
\end{aligned}
$$

so that

$$
S \approx 0.09 N_{\mathrm{D}} N_{\mathrm{TC}}
$$

Note that the value of $S$ does not depend on the value of the width to mass ratio

$$
\xi \equiv \frac{\Gamma_{\mathrm{V}}}{m_{\mathrm{V}}}=\frac{\Gamma_{\mathrm{A}}}{m_{\mathrm{A}}} .
$$

However, the spectral function and the $s$ dependence of the large momentum expansion coefficients do. In Fig. 2, we show the $\xi$-dependence of $\operatorname{Im} \mathcal{F}(s)$. In the limit $\xi \rightarrow+0$, these functions reduce to the well known $\delta$-function (vector-meson dominance) model, and the large momentum expansion coefficients have no $s$-dependence. In the other limit where $\xi \approx 1$, we obtain a smooth function reminiscent of the perturbative case, and the $s$-dependence of the expansion coefficients is large. In the following, we fix $\xi$ to the large- $N$ value

$$
\xi=\frac{\Gamma_{\mathrm{V}}}{m_{\mathrm{V}}} \approx \frac{\Gamma_{\rho}}{m_{\rho}} \approx 0.2
$$


Table 2: The $S$-parameter for the Breit-Wigner model function. The cutoffs are $s_{0}=0.2 m_{V}^{2}$ and $R=5 m_{V}^{2}$. The dimensionless parameters are $r=0.4$, $\xi=0.2$. The exact value of $S$ is 0.257 . (Since the $S_{\mathrm{IR}}=0.016$ correction is not included here, the results should be compared with 0.241).

\begin{tabular}{ccrcrr}
\hline \hline$N$ & $M$ & $S_{\mathrm{ACD}}$ & $S_{N, M}=S_{\mathrm{ACD}}+\Delta_{\mathrm{AC}}$ & $\Delta_{\text {fit }}$ & $\Delta_{\mathrm{tr}}$ \\
\hline \hline 3 & 2 & 0.657 & 0.557 & -0.057 & -0.244 \\
& 3 & 0.365 & 0.102 & & 0.211 \\
& 4 & 0.375 & 0.343 & & -0.030 \\
& 5 & 0.375 & 0.305 & & 0.009 \\
\hline 4 & 2 & 0.838 & 0.836 & -0.006 & -0.573 \\
& 3 & -0.063 & -0.445 & & 0.708 \\
& 4 & 0.009 & 0.624 & & -0.361 \\
& 5 & 0.118 & 0.183 & & 0.080 \\
& 6 & 0.118 & 0.244 & & 0.019 \\
\hline 5 & 3 & -1.083 & -1.578 & 0.014 & 1.821 \\
& 4 & -0.811 & 1.604 & & -1.361 \\
& 5 & 0.093 & 0.402 & & 0.645 \\
& 6 & -0.230 & 0.327 & & -0.085 \\
& 7 & -0.230 & 0.238 & & 0.005 \\
\hline 8 & 7 & 11.687 & -6.149 & 0.000 & 6.406 \\
& 8 & -3.111 & 2.397 & & -2.141 \\
& 10 & -1.195 & 0.028 & & 0.234 \\
\hline \hline
\end{tabular}

which is in between the two extremes.

The large momentum expansion of our model function is given by

$$
\mathcal{F}(s)=\frac{f_{\mathrm{TC}}^{2}}{s} \sum_{n=0}^{\infty} \frac{(-1)^{n} m_{\mathrm{V}}^{2 n}}{s^{n}}\left[X_{2 n}(r) U_{2 n}(\xi / 2)-i \frac{m_{\mathrm{V}}}{\sqrt{s}} X_{2 n+1}(r) U_{2 n+1}(\xi / 2)\right]
$$

\footnotetext{
${ }^{1}$ Actually, the integral for the second Weinberg sum rule does not converge for this model. We therefore impose the equivalent constraint that the $1 / Q^{4}$ term vanishes in the OPE for $\mathcal{F}\left(-Q^{2}\right)$.
} 
where $X_{n}(r)$ is defined as

$$
X_{n}(r) \equiv \frac{1-r^{1-\frac{n}{2}}}{1-r}
$$

while $U_{n}(\cos \theta)=\sin (n+1) \theta / \sin \theta$ are the Chebyshev polynomials of the second kind. Note that in the limit $\xi=0$, the $s$-dependence of the coefficients vanishes because $U_{2 n+1}(0)=0$.

We have a slight problem with the choice of fit interval since the branch cut of this model begins at $s=0$ but the IR cutoff $s_{0}$ must be kept nonzero. We will therefore choose $s_{0}$ to be well below the peak of the techni- $\rho$ resonance but non-zero. The approximate form of the spectral function below $s_{0}$ is

$$
-\operatorname{Im} \mathcal{F}(s) \approx\left(\frac{f_{\mathrm{V}}^{2}}{m_{\mathrm{V}}^{3}}-\frac{f_{\mathrm{A}}^{2}}{m_{\mathrm{A}}^{3}}\right) \xi \sqrt{s}=\frac{\xi f_{\mathrm{TC}}^{2} X_{-3}(r) \sqrt{s}}{m_{\mathrm{V}}^{3}} .
$$

This can be used to calculate analytically the contribution from the interval $\left[0, s_{0}\right]$ :

$$
S_{\mathrm{IR}}\left(s_{0}\right)=-4 \int_{0}^{s_{0}} \frac{d s}{s} \operatorname{Im} \mathcal{F}(s) \approx \frac{8 \xi f_{\mathrm{TC}}^{2} X_{-3}(r) \sqrt{s_{0}}}{m_{\mathrm{V}}^{3}} .
$$

The ACD technique is employed for estimating the contribution coming from the interval $\left[s_{0}, R\right]$. We must modify the large momentum expansion of $\mathcal{F}(s)$ slightly to subtract out the contribution of the interval $\left[0, s_{0}\right]$ :

$$
\mathcal{F}(s) \Longrightarrow \mathcal{F}(s)-\delta \mathcal{F}_{\mathrm{IR}}(s)
$$

where

$$
\begin{aligned}
\delta \mathcal{F}_{\mathrm{IR}}\left(-Q^{2}\right) & =\frac{1}{\pi} \int_{0}^{s_{0}} d s \frac{\operatorname{Im} \mathcal{F}(s)}{s+Q^{2}} \\
& \approx\left[\frac{\xi f_{\mathrm{TC}}^{2} X_{-3}(r) \sqrt{s_{0}}}{\pi m_{\mathrm{V}}^{3}}\right] \sum_{m=1}^{\infty} \frac{(-1)^{m}}{\left(m+\frac{1}{2}\right)} \frac{s_{0}^{m}}{Q^{2 m}}
\end{aligned}
$$

and our estimate of $S$ will be given by

$$
S \approx S_{\mathrm{IR}}\left(s_{0}\right)+S_{\mathrm{ACD}}\left(s_{0}, R\right)
$$

For our present analysis, we used the values $s_{0} / m_{\mathrm{V}}^{2}=0.2$, and $R / m_{\mathrm{V}}^{2}=5$.

Using these numbers and expressions, we calculated $S_{\mathrm{ACD}}$ and the three errors $\Delta_{\mathrm{AC}}, \Delta_{\mathrm{tr}}$, and $\Delta_{\text {fit }}$ for the case $N_{\mathrm{D}}=1, N_{\mathrm{TC}}=3$. The results are 
shown on Table 2. In this case, not only did the $S_{\mathrm{ACD}}$ approximation fail, but even the inclusion of the $s$-dependence did not give accurate and stable results. The reason is that the truncation error is oscillatory and converges too slowly. It will eventually converge since the large momentum expansion is convergent for this model, but not before $M$ becomes impractically large; in a realistic application one would have to truncate the OPE at $M=3$ or 4 .

\section{Conclusions}

We have shown that the ACD technique employed by Sundrum and Hsu in estimating the $S$-parameter is not completely reliable. We find that the neglect of the $s$-dependence of OPE coefficients is not a good approximation. In an example that we have analyzed we find that the truncation error is also not controllable, even if the spectral function is exactly known. This brings into doubt the utility of the ACD method in estimating the $S$ parameter in complicated theories like walking technicolor.

\section{Acknowledgments}

We would like to thank R. Sundrum for helpful discussions. S. R. I. and L. C. R. W. were supported in part by the U.S. Department of Energy under the grant \#DE-FG02-84ER40153.

\section{References}

[1] N. F. Nasrallah, N. A. Papadopoulos and K. Schilcher, Phys. Lett. B113 (1982) 61; B126 (1983) 379; Z. Phys. 16 (1983) 323. N. A. Papadopoulos, J. A. Peñarocha, F. Scheck and K. Schilcher, Phys. Lett. B149 (1984) 213; Nucl. Phys. B258 (1985) 1.

[2] R. Sundrum and S. D. H. Hsu, Nucl. Phys. B391 (1993) 127.

[3] B. Holdom, Phys. Lett. B150 (1985) 301, T. Appelquist, D. Karabali, and L. C. R. Wijewardhana, Phys. Rev. Lett. 57 (1986) 957; 
T. Appelquist and L. C. R. Wijewardhana, Phys. Rev. D35 (1987) 774, Phys. Rev. D36 (1987) 568;

K. Yamawaki, M. Bando and K. Matumoto, Phys. Rev. Lett. 56 (1986) 1335.

[4] M. E. Peskin and T. Takeuchi, Phys. Rev. Lett. 65 (1990) 964; Phys. Rev. D46 (1992) 381;

T. Takeuchi, in the Proceedings of the International Workshop on Electroweak Symmetry Breaking, Hiroshima, Japan, November 1991, edited by W. A. Bardeen, J. Kodaira, and T. Muta (World Scientific, Singapore, 1992).

The $S$ parameter has also been estimated by employing low energy effective Lagragian techniques by:

B. Holdom and J. Terning, Phys. Lett. B247 (1990) 88, M. Golden and L. Randall, Nucl. Phys. B361 (1991) 3.

[5] M. Harada and Y. Yoshida, Phys. Rev. D50 (1994) 6902.

[6] I. Caprini and C. Verzegnassi, Il Nuovo Cim. 80A (1984) 187.

[7] M. A. Shifman, A. I. Vainshtein, and V. I. Zakharov, Nucl. Phys. B147 (1979) 385;

V. A. Novikov, M. A. Shifman, A. I. Vainshtein, and V. I. Zakharov, Fortschr. Phys. 32 (1984) 585; Nucl. Phys. B249 (1985) 445.

[8] L. C. Goonetileke, S. R. Ignjatović, T. Takeuchi and

L. C. R. Wijewardhana (in preparation).

[9] S. Weinberg, Phys. Rev. Lett. 18 (1967) 507. 